\title{
Connection Oriented Isochronous Services in a DQDB Network: Specification of a Service-protocol pair and a Bandwidth Allocation Scheme*
}

\author{
G. Juanole, R. L. R. Carmo ${ }^{\dagger}$ and F. Vasques ${ }^{\ddagger}$ \\ $L A A S d u C N R S$ \\ 7, Avenue du Colonel Roche - 31077 Toulouse Cedex FRANCE \\ email: $\{$ juanole, carmo,vasques\}@laas.fr
}

\begin{abstract}
This paper addresses the problem of transmitting real-time isochronous traffic in a DQDB network using the Pre-Arbitrated (PA) access. In the DQDB standard, a framework is defined to provide connection-oriented isochronous services using PA access. In this framework, mechanisms as a bandwidth allocation scheme, to provide guaranteed bandwidth to real-time applications, are not provided. Furthermore only guidelines of a signaling protocol to establish/release isochronous connections are given.

The goal of this paper is twofold: to specify a service-protocol pair for establishing/releasing isochronous connections and to define a bandwidth allocation scheme for a set of isochronous connections to guarantee a hard real-time isochronous service. The bandwidth allocation scheme consists of an off-line centralized scheduling algorithm and an on-line mode change algorithm which allows to take into account load changes.
\end{abstract}

\section{Keywords}

High speed networks, Petri net based models, real-time scheduling.

*This work has been partially supported by CNET-FT under grant $n^{\circ} 92-1 \mathrm{~B}-178$ as part of CESAME, a CNET-CNRS collaborative project on the Formal Design of High Speed Multimedia Systems

${ }^{\dagger}$ Financially supported by $\mathrm{CNPq}$, Brazil

${ }^{\ddagger}$ Financially supported by a JNICT grant ( $n^{\circ}$ BD 1278/91) 


\section{INTRODUCTION}

The DQDB protocol is a Medium Access Control (MAC) protocol which has been defined by IEEE as a standard (IEEE802.6) for Metropolitan Area Networks (MAN) [IEE90]. Two access controls are specified: the Pre-Arbitrated (PA) for Isochronous services (ex: video, voice) and the Queue Arbitrated (QA) for Asynchronous services (ex: data).

The QA access mechanisms are detailed in the standard [IEE90] and the study, the analysis and the evaluation of these mechanisms have been made in numerous works [CJ94], [MB92], [TGS91]. On the other hand, mechanisms to provide a real-time isochronous service, based on the PA access, are not described in the standard.

Today, in the increasing context of distributed multimedia applications (applications integrating video, voice and text) which are real-time applications based on high speed networks, it is essential to specify PA mechanisms for providing real-time isochronous services in a DQDB network. This is precisely the goal of this paper.

This paper includes three sections. The second section presents a proposal in terms, at first, of a service-protocol pair for requesting bandwidth allocation and releasing bandwidth for connection-oriented isochronous services, and second, of a bandwidth allocation scheme for a set of connections, which can change during the time. Note, concerning the bandwidth allocation scheme, that we benefit from well-known results in the area of centralized scheduling [LL73], which are suitable for our problem. The third section is concerned by the formal specification and verification of the suggested service-protocol pair. The formal modeling is based on Petri Nets which are a suitable formalism for the specification of communication mechanisms (parallelism, synchronization). In this way, we can have confidence in the suggested specification. The fourth section specifies a bandwidth allocation scheme which allows, at first, to schedule a set of real-time isochronous connections, and second, to take into account load changes (called mode changes) by guaranteeing correctness and responsiveness.

\section{PROPOSAL}

We now present the main features of our proposal in terms of an architecture for the PA access; a service-protocol pair for requesting a bandwidth allocation and releasing an allocated bandwidth (primitives, PDUs and exchange scheme); and a bandwidth allocation scheme.

\section{Architecture for the PA access}

In the standard [IEE90], a framework is defined in order to support a connection-oriented isochronous service. This framework is composed of the following modules:

- in each node, the MAC entity includes, in particular, the following two modules: an Isochronous Convergence Function (ICF) module and a PA module.

- the ICF module must provide, at first, the bandwidth allocation and release (by means of a connection establishment/disconnection service/protocol pair) and, second, during the time where the bandwidth is allocated, the adaptation between the rates of the application layer and the PA service. Concerning the 
adaptation: its main function on the emission side, is buffering the Information Units (IUs) coming from the application, fragmenting the IUs at the size of the PA segment payload field ( 48 bytes) of a slot and sending this field to the PA module; its main function on the receiving side, is receiving the PA segment payload fields from the PA module, reassembling these fields into IUs and sending these IUs to the application layer.

- the PA module, on the one hand, provides a service to the ICF module, receiving and sending the PA segment payload field and, on the other hand, controls the medium access, i.e. writing and reading the slot ( 53 bytes).

- in one node, there must be a Signaling Termination (ST) module which makes the management of the signaling protocol for establishing connections (i.e. requesting bandwidth allocation).

- in one node, there must be a Bandwidth Manager (BM) module which decides, when it is requested by the ST module whether bandwidth can be or cannot be allocated to the connection.

In the standard neither the location of the ST and BM modules nor a precise exchange scheme are specified. We propose the architecture which is represented on the figure 1.

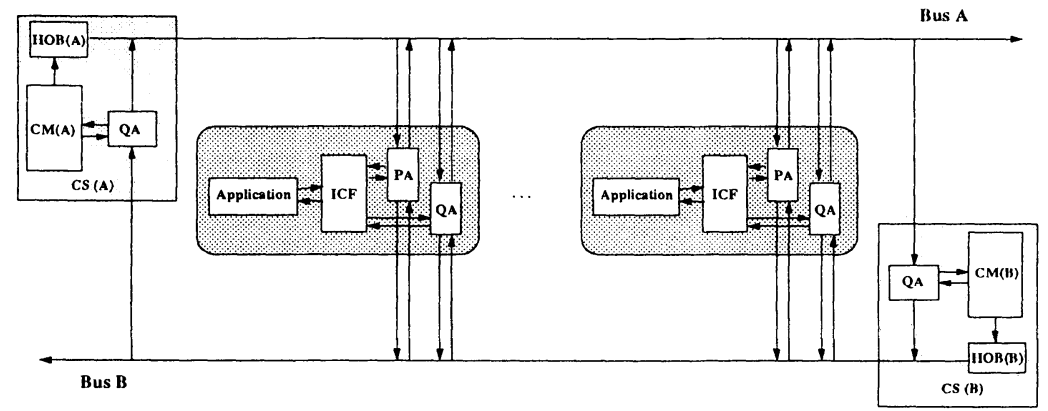

Figure 1: Architecture for the PA access.

- we put the ST and BM modules relative to each bus in a same module called CM (Control Module). The CM module is located in the node at the head of the bus, i.e. the node which includes the HOB module. In this way, when the CM module will decide to allocate bandwidth to a connection, it will order the HOB module to put a PA mark in the slot type field of a generated slot. The node at the head of a bus is globally called a Control Station (CS) which includes QA, CM, and HOB modules. Hence, we have a CS(A) station at the head of the bus A and a CS(B) station at the head of the bus B. Note that as we are not considering applications in the control stations, they do not need a PA module. 
- in the other nodes, i.e. the nodes where we have user applications with real-time isochronous service requirements, we have represented an application module which uses the service of the ICF module which itself can use the QA and PA modules. The QA module is used for requesting a bandwidth allocation and releasing an allocated bandwidth. Once the bandwidth has been allocated, the ICF module uses the PA module for transferring the periodic data.

\section{Service-protocol pair}

We then consider the service-protocol pair which concerns the layer composed of the ICF modules, in the nodes where we have applications and of the modules $\mathrm{CM}(\mathrm{A})$ and $\mathrm{CM}(\mathrm{B})$ respectively in the nodes $\mathrm{CS}(\mathrm{A})$ and $\mathrm{CS}(\mathrm{B})$. The role of the service-protocol pair is to provide the allocation of a requested bandwidth to the application entities and to release this bandwidth.

We now present the basic ideas for specifying such a service-protocol pair: exchange scheme, primitives between application entities and ICF entities, PDUs between ICFs, $\mathrm{CM}(\mathrm{A})$ and $\mathrm{CM}(\mathrm{B})$. We do not consider here the underlying QA mechanism.

\section{Specification context}

\section{Hypothesis}

- the application entities require real-time isochronous services with a bidirectional exchange of traffic, then they need bandwidth allocation on the bus $\mathrm{A}(\mathrm{CM}(\mathrm{A})$ will do that) and on the bus B (CM(B) will do that).

- the associations between two application entities for the bandwidth allocation phase, are governed by the concept Initiator-Responder. Furthermore, each application entity define its period.

- the associations between two application entities for the bandwidth release phase are symmetrical, i.e. any entity can initiates the bandwidth release phase.

\section{Basic ideas}

\section{Bandwidth allocation phase}

The ICF module, associated to the initiator application entity, begins this phase sending a message to the CM module in the CS node which controls the bus where the responder side is downstream. It is in this bus that the initiator side will send its IUs to the responder side. The responder side will send its IUs to the initiator side on the other bus. This $C M$ module, in particular, has the leading role during the bandwidth allocation phase.

\section{Bandwidth release phase}

We adopt the concept of no graceful disconnection. The ICF module requesting a disconnection do not wait the response of the other ICF, to consider that the connection is released. On the other hand, each ICF waits the disconnection messages of the two CM modules to consider that the connection is released. 


\section{The bandwidth allocation phase}

We define, for each bus, the concepts of locked bandwidth and allocated bandwidth. These concepts allow to specify the exchange scheme between the ICF and the CM modules. Call ICF(I) and ICF(R) the ICF modules in respectively the initiator and responder side, and $\mathrm{CM}(\mathrm{L})$ and $\mathrm{CM}(\bar{L})$ the $\mathrm{CM}$ modules which have respectively the Leading role and Not the Leading role.

We propose the following exchange scheme:

1. $\mathrm{ICF}(\mathrm{I})$ sends to $\mathrm{CM}(\mathrm{L})$ its request for a bidirectional communication between the initiator address and the responder address.

2. $\mathrm{CM}(\mathrm{L})$, if it agrees (available bandwidth on its bus), locks bandwidth and propagates the request of ICF(I) with its positive answer and the identifier of the VCI (virtual channel identifier) that it forecasts for this isochronous connection.

3. $\operatorname{ICF}(\mathrm{I})$ sees the answer of $\mathrm{CM}(\mathrm{L}) . \operatorname{ICF}(\mathrm{R})$ and $\mathrm{CM}(\bar{L})$ know the request of $\operatorname{ICF}(\mathrm{I})$ and the answer of $\mathrm{CM}(\mathrm{L})$.

4. $\operatorname{ICF}(R)$ if it agrees to establish a connection with $\mathrm{ICF}(\mathrm{I})$, will transmit its agreement to $\mathrm{CM}(\bar{L})$ on the bus controlled by $\mathrm{CM}(\mathrm{L})$.

5. $\mathrm{CM}(\bar{L})$ when it receives the positive answer of $\mathrm{ICF}(\mathrm{R})$ and if it agrees (available bandwidth on its bus), locks bandwidth and transmits, on its bus, to ICF(R), ICF(I) and $\mathrm{CM}(\mathrm{L})$, its positive answer and the identifier of the $\mathrm{VCI}$ that it forecasts for this isochronous connection.

6. When $\mathrm{CM}(\mathrm{L})$ receives the agreement of $\operatorname{CM}(\bar{L})$, it has the global knowledge that all the different partners agree. Then it sends on its bus, the connection order to $\operatorname{ICF}(\mathrm{I}), \operatorname{ICF}(\mathrm{R})$ and $\mathrm{CM}(\bar{L})$.

Remark: We will consider that the different entities $\mathrm{CM}(\mathrm{L}), \mathrm{CM}(\bar{L}), \operatorname{ICF}(\mathrm{R})$ can refuse

\section{The bandwidth release phase}

As there is not a leading module in this phase, we maintain the notation $\mathrm{CM}(\mathrm{L})$ and $\mathrm{CM}(\bar{L})$ used in the bandwidth allocation phase.

Consider that ICF(I) starts the bandwidth release phase. We propose the following scheme:

1. $\mathrm{ICF}(\mathrm{I})$ sends to $\mathrm{CM}(\mathrm{L})$ its request for releasing the bandwidth.

2. $\mathrm{CM}(\mathrm{L})$ transmits to $\operatorname{ICF}(\mathrm{I}), \operatorname{ICF}(\mathrm{R})$ and $\mathrm{CM}(\bar{L})$, a message informing that it releases the VCI allocated on its bus for the communication between ICF(I) and ICF(R).

3. $\mathrm{CM}(\bar{L})$, after reception of the message coming from $\mathrm{CM}(\mathrm{L})$ transmits to $\operatorname{ICF}(\mathrm{R})$, $\mathrm{ICF}(\mathrm{I})$, and $\mathrm{CM}(\mathrm{L})$ a message informing that it releases the VCI allocated on the bus for the communication between ICF (R) and ICF(I). When ICF (R), ICF(I) and $\mathrm{CM}(\mathrm{L})$ receive this message, they consider that the communication is terminated. They have the knowledge that the bandwidth is no more allocated. 
Remark: In the case of a ICF $(R)$ request for releasing the bandwidth, we have the same scheme with the role of $\operatorname{ICF}(\mathrm{I})$ and $\operatorname{ICF}(\mathrm{R})$ and the role of $\mathrm{CM}(\mathrm{L})$ and $\mathrm{CM}(\bar{L})$ reversed.

\section{Primitives and PDUs}

Before describing the primitives and PDUs, we give some notation about the parameters: $a d d r I$ and $a d d r R$ mean respectively the address of the initiator and the address of the responder; bidir means bidirectional exchange; per $I$ is the period of the traffic generated by the initiator and per $R$ is the period of the traffic generated by the responder; $\operatorname{VCI}(A)$ and $V C I(B)$ mean respectively the identifier of a virtual connection on the bus $A$ and the identifier of a virtual connection on the bus $\mathrm{B}$.

\section{Requesting bandwidth}

\section{Primitives between the application and the $\operatorname{ICF}(\mathrm{I})$}

- open_request (addr I, addr R, bidir, per I): the application requests a connection

- open_conf(+) (addr I, addr R, bidir, per I, per R): ICF(I) confirms the connection

- open_conf(-) (addr I, addr R, bidir, per I): ICF(I) informs that the connection is refused

\section{Primitives between the application and the $\operatorname{ICF}(\mathrm{R})$}

- open_ind (addr I, addr R, bidir, per I): ICF(R) informs that a connection is requested

- open_resp $(+)$ (addr I, addr R, bidir, per I, per R): the application accepts the connection

- open_resp(-) (addr I, addr R, bidir, per I): the application refuse the connection

- est_ind $(+)$ (addr I, addr R, bidir, per I, per R): ICF (R) informs that the connection is established

- est_ind(-) (addr I, addr R, bidir, per I, per R): ICF(R) informs that the connection is refused

\section{PDUs for requesting bandwidth allocation and accepting this request}

- set_up (addr I, addr R, bidir, per I): request from ICF(I)

- set_up_ack_A (addr I, addr R, bidir, per I, VCI $(A)$ ): agreement of CM(A)

- set_up_ack (addr I, addr R, bidir, per R): agreement of ICF(R)

- set_up_ack_B (addr I, addr R, bidir, per R, VCI(B)): agreement of CM(B)

- connect (addr I, addr R, bidir, per I, per R, VCI(A), VCI(B)): connection order given by $\mathrm{CM}(\mathrm{A})$

\section{PDUs for refusing the bandwidth allocation request}

- set_up_nack_A (addr I, addr R, bidir, per I): refusal of CS(A)

- set_up_nack_B (addr I, addr R, bidir, per R): refusal of CS(B)

- set_up_nack (addr I, addr R, bidir, per I): refusal of ICF(R) 


\section{Releasing bandwidth}

\section{Primitives between the application and the ICF(I)}

- rel_req (addr I, addr R, bidir, per I): ICF(I) requests the disconnection

- relind (addr I, addr $\mathrm{R}$, bidir, per $\mathrm{I}$ ): ICF(I) informs that the connection is released

\section{Primitives between the application and the $\operatorname{ICF}(\mathrm{R})$}

- rel_req (addr I, addr R, bidir, per $\mathrm{R})$ : $\operatorname{ICF}(\mathrm{R})$ requests the disconnection

- rel_ind (addr I, addr R, bidir, per $R$ ): $I C F(R)$ informs that the connection is released

\section{PDUs}

- disconnect (addr I, addr $\mathrm{R}, \mathrm{VCI}(\mathrm{A})$ ): request from $\mathrm{ICF}(\mathrm{I})$

- disconnect (addr I, addr R, VCI(B)): request from ICF $(\mathrm{R})$

- disconnect_A (addr I, addr R, VCI(A)): release of VCI(A) by $\mathrm{CM}(\mathrm{A})$

- disconnect_B (addr I, addr R, VCI(B)): release of VCI(B) by $\mathrm{CM}(\mathrm{B})$

\section{Bandwidth allocation scheme}

The number of applications which require real-time isochronous services changes dynamically. We have continuously requests for opening and releasing connections. In order to guarantee a real-time isochronous service in a high speed dynamic environment, we propose the following strategy:

- as a DQDB network is a high speed network, it is technically impossible (at least nowadays) to compute on-line bandwidth allocation for the periodic traffic (timeline) and to still satisfy the application timing requirements. Therefore the time-line must be computed before its starting up: selection of an off-line scheduling algorithm.

- the behavior of the end users requests for opening/releasing isochronous connections is absolutely unpredictable. Thus, means for changing on-line the operation mode must be provided: selection of an on-line mode change algorithm.

These two algorithms are implemented in the module CM of the nodes CS(A) and $\mathrm{CS}(\mathrm{B})$.

The off-line scheduling algorithm, which is based on the well-known Rate Monotonic (RM) algorithm [LL73] consists, at first, of a feasibility test (can a new connection request be scheduled by always satisfying the real-time requirements?) and second, if the feasibility test is positive, of a time-line generation algorithm (new bus slot allocation).

The on-line mode change algorithm decides the time when the time-line changing operation can be executed, guaranteeing a minimum responsiveness level to the system mode change. 


\section{FORMAL MODELING AND VERIFICATION OF THE SERVICE PROTOCOL PAIR}

\section{Methodology}

The formal analysis is based on the formal model labeled Petri nets [Llo90]. These nets are an extension of classical Petri nets [BRA83]. One label has the format condition and/or action : the conditions are the reception of messages (noted ?M) and the actions are the sending of messages (noted !M).

A symbolic interpreter, called PIPN (Prolog Interpreter Petri Nets) [Llo90], which allows the execution of the specifications has been developed in LAAS and Verilog Society. Prolog predicates are used for describing the formal model. The interpreter allows composition of nets; composition being derived from transition merging: a transition with a label !X can be merged with a transition with a label ?M.

The interpreter can perform the following verification operations: exploration of the space of reachable states (reachability analysis which gives the marking graph); projection methods on the marking graph (an automaton) in order to obtain abstract views with respect to a set of events and/or states.

\section{Modeling}

\section{Hypothesis}

We consider that the initiator node is upstream on the bus A, i.e. it is on the left on the figure 1. Thus, the CM module which has the leading role is the $\mathrm{CM}(\mathrm{A})$.

\section{Local models}

The Petri net models of the ICF(I), ICF $(R), C M(A)$ and $C M(B)$ are represented respectively in the figures $2,3,4$ and 5 . The transitions are only labeled with the names of the primitives and/or PDUs; the parameters are not represented.

Let us briefly comment only the ICF(I) and ICF (R) models. The models of CM(A) and $\mathrm{CM}(\mathrm{B})$ can easily be understood after this presentation.

\section{ICF(I) model: figure 2}

The transition sequence $t_{1}, t_{2}, t_{3}, t_{4}$ represents a successful bandwidth allocation phase.

The unsuccessful allocation phase is represented by several sequences: sequence $t_{1}, t_{8}$ (refusal of $\mathrm{CM}(\mathrm{A})$ ); sequence $t_{1}, t_{2}, t_{9}, t_{10}$ (refusal of $\mathrm{CM}(\mathrm{B})$ ); sequence $t_{1}, t_{2}, t_{11}, t_{12}$ (refusal of $\operatorname{ICF}(\mathrm{R}))$.

The bandwidth release phase is represented by several sequences: sequence $\left(t_{5}, t_{6}, t_{7}\right)$ and $\left(t_{5}, t_{15}, t_{14}\right)$ (ICF (I) requests the disconnection); $t_{13}, t_{14}$ (ICF (R) requests the disconnection).

\section{$\operatorname{ICF}(\mathrm{R})$ model: figure 3}

The transition sequence $t_{1}, t_{2}, t_{3}, t_{4}$ represents a successful bandwidth allocation phase. 


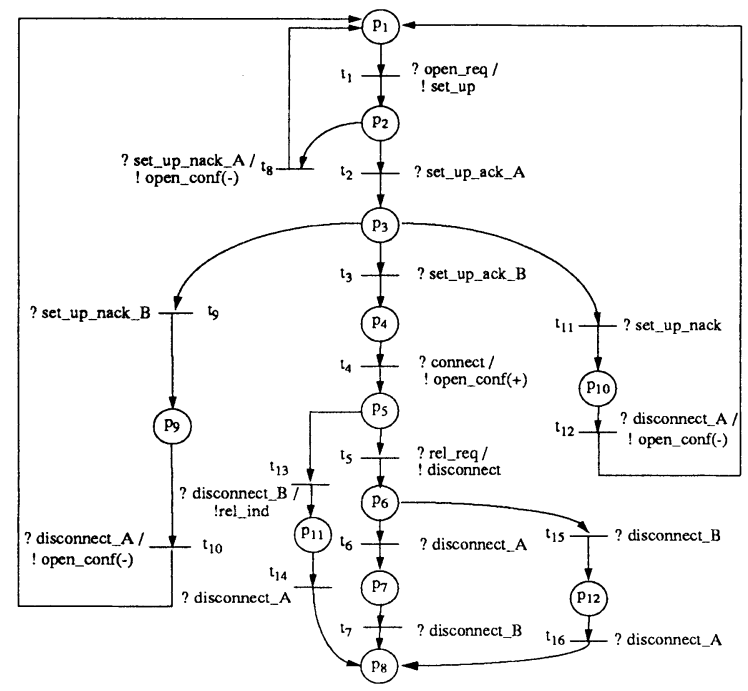

Figure 2: ICF (I) model.

The no successful allocation phase is represented by one transition $t_{8}$ (refusal of $\mathrm{CM}(\mathrm{A}))$ and two sequences: sequence $t_{1}, t_{11}, t_{12}, t_{13}$ (refusal of $\mathrm{I}\left(\mathrm{H} \cdot \mathrm{H}(\mathrm{R})\right.$ ); sequence $t_{1}, t_{2}, t_{9}, t_{10}$ (refusal of $\mathrm{CM}(\mathrm{B}))$.

The bandwidth release phase is represented by several sequences: sequence $\left(t_{5}, t_{6}, t_{7}\right)$ and $\left(t_{5}, t_{16}, t_{17}\right)\left(\mathrm{ICF}(\mathrm{R})\right.$ requests the disconnection); $t_{14}, t_{15}$ (ICF(I) requests the disconnection).

\section{Global model}

The Global model is got by interconnecting the four models described in the previous section through a medium which is considered without losses.

\section{Verification}

Two kinds of properties have been verified on the global model: general properties (i.e. not dependent on the mission) and specific properties (i.e. dependent on the mission).

\section{Global properties}

The marking graph (number of states) is bounded (5927 markings), live (all the transitions are fired). It proofs that this specification can be implemented. 


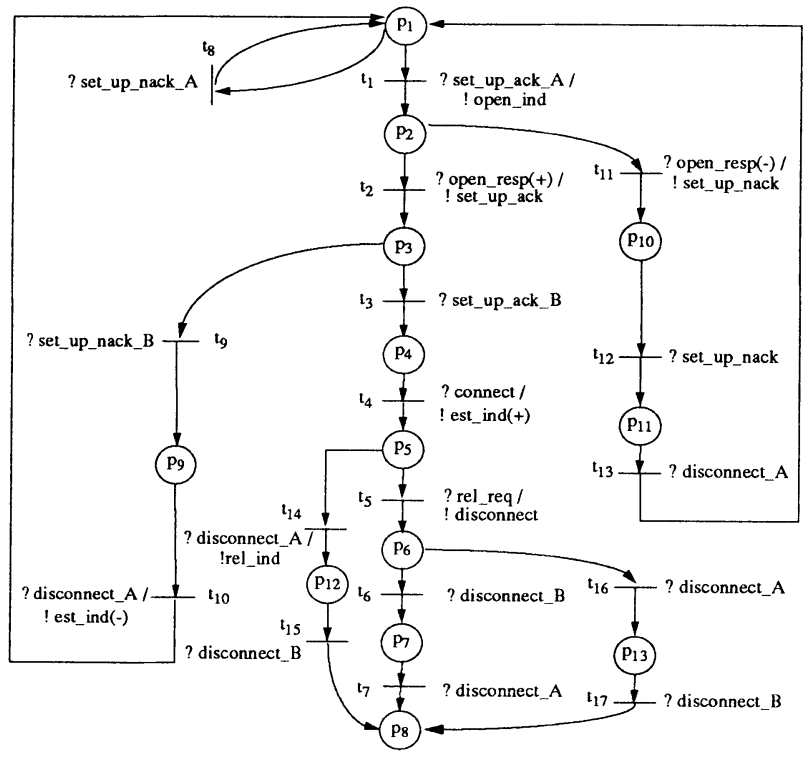

Figure 3: $\operatorname{ICF}(\mathrm{R})$ model.

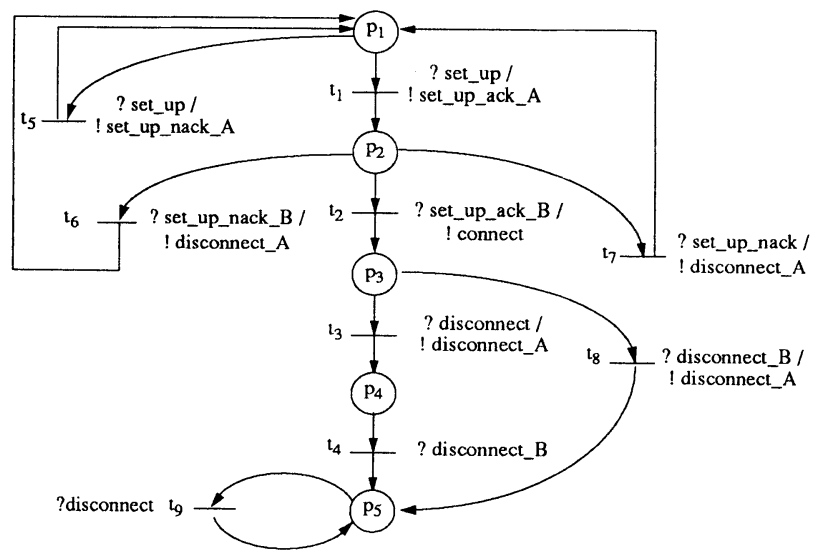

Figure 4: CM(A) model. 


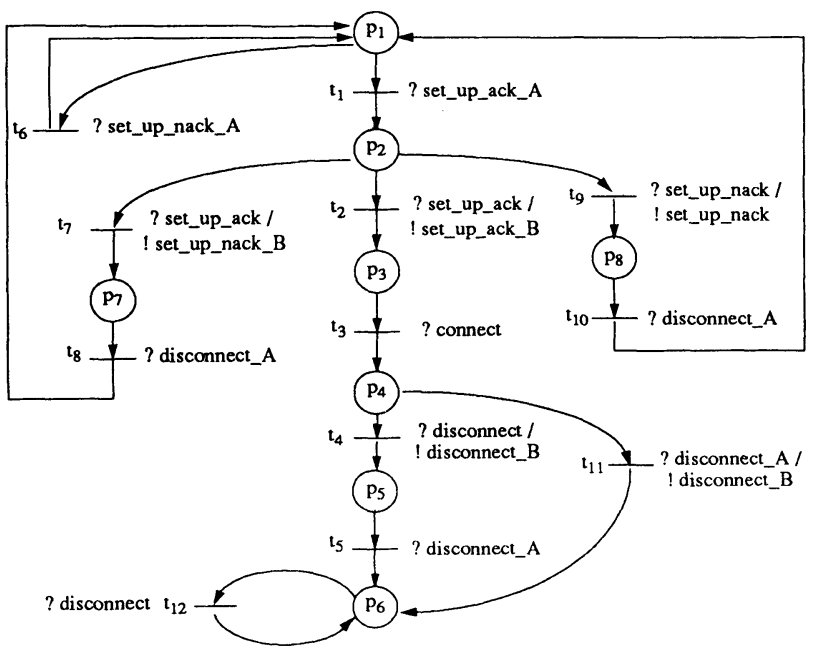

Figure 5: CM(B) model.

\section{Specific properties}

The specific properties are got through a projection technique based on the observational equivalence [Mil80]. We can get abstract views representing the behavior of a system not only with respect to observable events but also to unobservable events which occurrence influence the trace of the observable ones.

Two kinds of abstract views were obtained: the abstract views of the local service provided by $\operatorname{ICF}(\mathrm{I})$ and $\operatorname{ICF}(\mathrm{R})$ and the abstract view of the global service provided by the ICF layer to the applications entities. We have made a projection on the service primitives (observable events). $C_{1}, C_{2}, \ldots, C_{n}$ are equivalent state classes.

\section{Local services: figure 6}

\section{Service at the initiator side (ICF(I))}

After the reception of the open_req by ICF(I), there are two unobservable events: $\tau_{1}$ represents the acceptance of $\mathrm{CM}(\mathrm{A}), \mathrm{ICF}(\mathrm{R})$ and $\mathrm{CM}(\mathrm{B})$ and $\tau_{2}$ represents the refusal of $\mathrm{CM}(\mathrm{A})$ or $\mathrm{ICF}(\mathrm{R})$ or $\mathrm{CM}(\mathrm{B})$. The sequence $C_{1}, C_{2}, C_{3}, C_{5}$ represents a successful bandwidth allocation phase and the sequence $C_{1}, C_{2}, C_{4}, C_{1}$ an unsuccessful one.

The sequence $C_{5}, C_{6}$ represents the bandwidth release phase when $\mathrm{ICF}(\mathrm{I})$ requests (rel_req) and when $\mathrm{ICF}(\mathrm{R})$ requests (rel_ind).

\section{Service at the responder side $(\mathrm{ICF}(\mathrm{R}))$}

After the acceptance of $\operatorname{ICF}(\mathrm{R})\left(C_{1}, C_{2}, C_{3}\right)$, there are two unobservable events: $\tau_{1}$ means the refusal of $\mathrm{CM}(\mathrm{B})$ and $\tau_{2}$ means the acceptance of $\mathrm{CM}(\mathrm{B})$. The sequence $C_{1}, C_{2}, C_{3}, C_{5}, C_{6}$ represents a successful bandwidth allocation phase and the sequences 
$C_{1}, C_{2}, C_{1}$ (ICF(R) refusal) and $C_{1}, C_{2}, C_{3}, C_{4}, C_{1}$ (CM(B) refusal) an unsuccessful one.

The sequence $C_{6}, C_{7}$ represents the bandwidth release phase when $\mathrm{ICF}(\mathrm{R})$ requests (rel_req) and when ICF(I) requests (rel_ind).

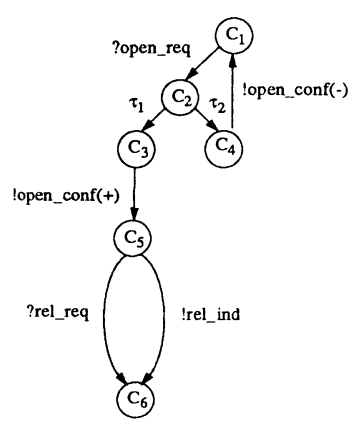

a- Service du côté appelant

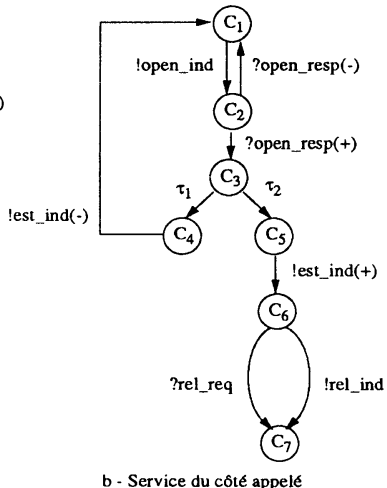

b- Service du cóté appelé

Figure 6: Local services.

\section{Global service: figure 7}

We notc respectively rel_req1, rel_ind 1 and rel_req 2 , rel_ind 2 the primitives to release bandwidth at the $\operatorname{ICF}(\mathrm{I})$ and at the $\operatorname{ICF}(\mathrm{R})$.

This figure illustrates the following points:

- after the reception of the open_req by $\operatorname{ICF}(\mathrm{I})\left(C_{1}, C_{2}\right)$, we have two unobservable events, $\tau_{1}$ and $\tau_{2}$, which have consequences for the future behavior: $\tau_{1}$ represents the acceptance of $\mathrm{CM}(\mathrm{A})$ and $\tau_{2}$ represents the refusal of $\mathrm{CM}(\mathrm{A})$.

- after the acceptance of $\operatorname{ICF}(\mathrm{R})$ (open_resp $(+)$ ), there are two possibilities: $\mathrm{MC}(\mathrm{B})$ accepts $\left(\tau_{3}\right)$ or $\mathrm{MC}(\mathrm{B})$ refuse $\left(\tau_{4}\right)$.

- the sequence $C_{1}, C_{2}, C_{3}, C_{5}, C_{6}, C_{7}, C_{9}, C_{10}, C_{12}, C_{13}, C_{14}, C_{15}, C_{16}$ represents a successful bandwidth allocation phase. The sequences $\left(C_{7}, C_{9}, C_{12}, C_{15}\right),\left(C_{7}, C_{9}, C_{13}, C_{15}\right)$, $\left(C_{7}, C_{10}, C_{13}, C_{16}\right)$ and $\left(C_{7}, C_{10}, C_{14}, C_{16}\right)$ represent the interleaving of the end of this phase in the ICF(I) and ICF (R) modules. Note that the release phase can start in the ICF(I) or in the $\operatorname{ICF}(\mathrm{R})$ before the termination of the allocation phase.

- the sequences $\left(C_{9}, C_{12}, C_{15}, C_{17}\right),\left(C_{13}, C_{15}, C_{17}\right),\left(C_{13}, C_{16}, C_{17}\right)$ and $\left(C_{10}, C_{14}, C_{16}, C_{17}\right)$ represent the bandwidth release phase. Note that passing from $C_{15}$ or $C_{16}$ to $C_{17}$ through the arc labeled with rel_req means the crossing of the PDUs of this phase. 
Finally we can say that the protocol which has been specified provides the expected service.

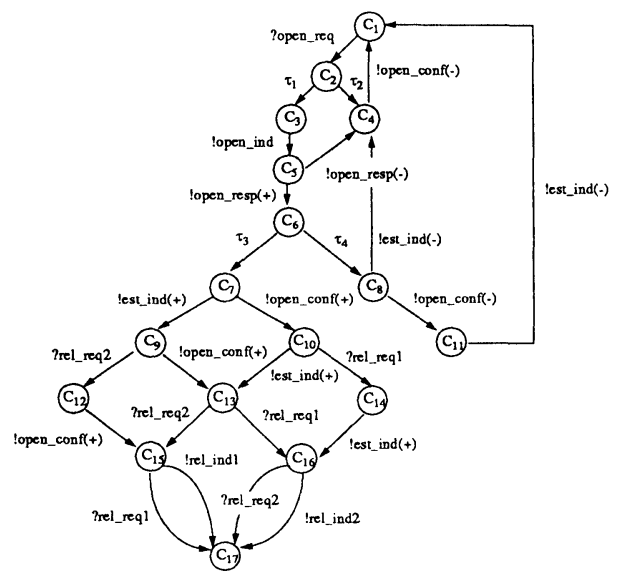

Figure 7: Global service.

\section{BANDWIDTH ALLOCATION SCHEME}

\section{The off-line scheduling algorithm}

\section{The Feasibility Test}

In order to make this test, we have, at first, to evaluate the bus utilization.

Consider a set of $\mathrm{n}$ connections $C_{i}$ with periods $P_{i} . P_{i}$ is the integer number of slots which separates two consecutive slots conveying data of the connection $C_{i}$. Then the bus utilization $\mathrm{U}$ is:

$$
\sum_{i=1}^{n} 1 / P_{i}
$$

As we are considering the use of the RM algorithm, the appropriated feasibility test is the following one:

$$
\sum_{i=1}^{n} 1 / P_{i} \leq n\left(2^{1 / n}-1\right)
$$

The limit of 4 when $n \rightarrow \infty$ is $69.3 \%$ which then keeps always bandwidth for the asynchronous traffic transmitted using QA access.

\section{The time-line generation}

The time-line generation algorithm provides the bus slot allocation for a previously defined periodic connection set. As we are considering the use of the RM algorithm, the slot 
allocation must comply with the following condition:

Slots are allocated first to connections with shorter periods.

The time-line is computed for the time-window $t_{l}, 0 \leq t_{l} \leq \operatorname{LCM}\left(P_{1}, P_{2}, P_{3}, \ldots, P_{n}\right)$. We call $\operatorname{LCM}\left(P_{1}, P_{2}, P_{3}, \ldots, P_{n}\right)$ the time-line cycle. The generation of the time-line is got by the execution of the following rules:

Rule 1 - Ordering connections.

$A$ list of all connections sorted in order of increasing period is generated, $P_{1} \leq P_{2} \ldots \leq$ $P_{j} \leq P_{j+1} \ldots \leq P_{n}$.

Rule 2 - Assignment of the slots to the connections. Slots are assigned first to connections with shorter periods for all the time-line cycle.

For each connection $C_{i}$ in the list, with $i=1,2, \ldots, n$ do: 1) $P_{i}$.

For $k=0$ to $\left(\left(L C M / P_{i}\right)-1\right)$ assign the first available slot of the interval $I, k P_{i} \leq I<(k+$

With the rule 2 , slots are assigned to a connection $C_{i}$ for every occurrence of $P_{i}$ in the time-line cycle. As connections are considered in the increasing order of their periods, it is guaranteed that connections with shorter periods always gain the access first. We note $P_{i}^{k}$ the $k^{t h}$ occurrence of the period $P_{i}$.

An example of the time-line is represented on the figure 8 . We have 3 connections: $C_{1}\left(P_{1}=4\right), C_{2}\left(P_{2}=6\right), C_{3}\left(P_{3}=12\right)$. Then the $\operatorname{LCM}\left(P_{1}, P_{2}, P_{3}\right)=12$.

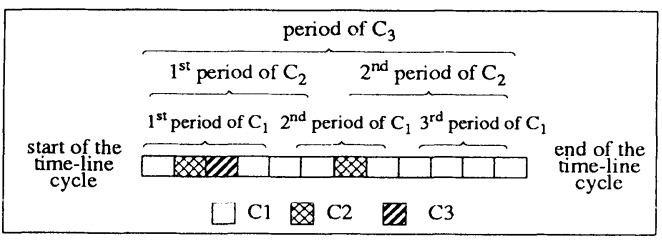

Figure 8: Time-line example.

\section{On-line mode change algorithm}

The goal of this algorithm is to provide the transition of a running time-line to a new timeline guaranteeing correctness on the slots generation. By correctness we mean that only and only one slot will be generated for every $P_{i}^{k}$ of every connection $C_{i}$ of the running timeline which still will be in the new time-line. The time-line transition must be transparent to these connections.

In order to present the algorithm, we give, at first, two examples to illustrate the correctness problem. Then we present some important concepts and we successively consider the cases of adding one connection and removing one connection.

\section{Examples}

\section{Example 1}

Consider the running time-line generated for a set of six connections $C_{1}, C_{2}, \ldots, C_{6}$ with periods $P_{1}=5, P_{2}=\ldots P_{6}=20$ and the new time-line which results from the addition of the connection $C_{7}$ with period $P_{7}=5$ (figures 9.a, 9.b). Suppose that the transition 
is made after the generation of the fourth slot in the running time-line. The slots will be generated as it is illustrated on the figure 9.c. We can see that one "extra" slot will be assigned to connection $C_{4}$ in this period, leading to a lack of correctness.

The assignment of an "extra" slot (slot duplication) for one connection $C_{i}$ in the koccurrence of the period $P_{i}$, results from the fact that the condition of the connection is different in the two time-lines when the transition is made. Taking the example of the figure 9 , the condition of $C_{4}$ at the running time-line is: the slot requested in $P_{4}^{1}$ have already been assigned; in the new time-line it is: the slot requested in $P_{4}^{1}$ has not yet been assigned.

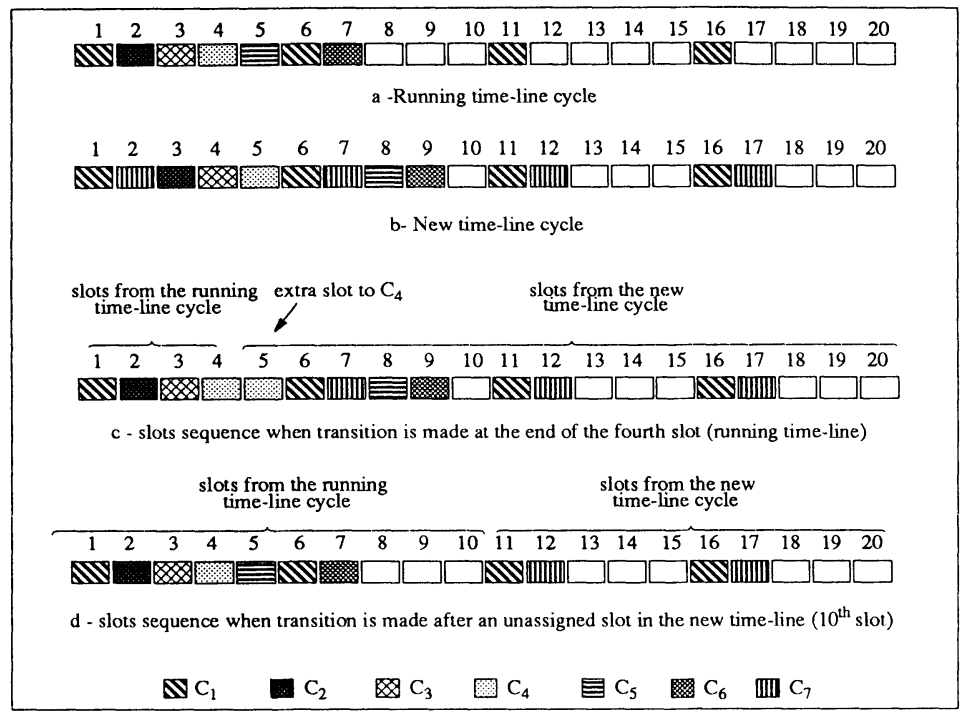

Figure 9: Time-line transition when a connection is added.

\section{Example 2}

Consider now the reverse of the previous example, with the same connection parameters. The new time-line and the running time-line of the previous case are now, respectively, the running time-line and the new time-line (figures 10.a, 10.b).

Suppose that the connection $C_{7}$ has to be removed from the running time-line. If the transition is made after the fourth slot generation in the running time-line, a slot is not assigned for connection $C_{4}$ for the period $P_{4}^{1}$ (slot loss) (figure 10.c). As in the previous case, the condition of $C_{4}$ is different in the time-lines: in the running time-line, the slot has not yet been assigned to $C_{4}$; in the new time-line, the slot has already been assigned to $C_{4}$.

\section{Conclusion}

These examples show that the correctness cannot be guaranteed when the time-line transition is made at any instant. 


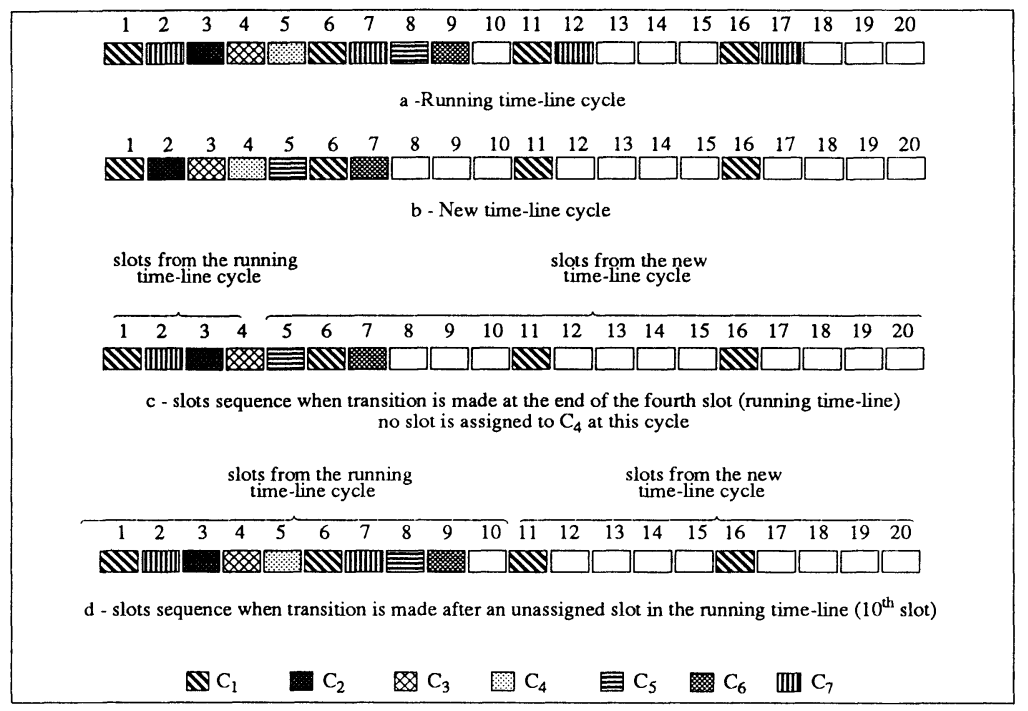

Figure 10: Time-line transition when a connection is removed.

\section{Concepts}

Two kinds of concepts must be introduced: concepts related to the time-line itself; and concepts related to the transition between a running time-line and a new time-line.

\section{Time-line concepts}

Definition 4.1 (Pending connection) $A$ connection $C_{i}$ is pending at the instant $t_{l}$ of the time-line, such that $k P_{i} \leq t_{l}<(k+1) P_{i}, k=0,1, \ldots,\left(L C M / P_{i}-1\right)$, if no slot has yet been assigned for this connection in the $k^{\text {th }}$ occurrence of the period.

Lemma 4.1 If $t_{l}$ is the starting instant of an unassigned slot, then there is no pending connection at $t_{l}$.

\section{Proof}

Assume that there is a pending connection $C_{i}$ at $t_{l}, k P_{i} \leq t_{l}<(k+1) P_{i}, k=$ $0,1, \ldots,\left(L C M / P_{i}-1\right)$, and $t_{l}$ is the starting instant of an unassigned slot.

This contradicts the rule $\mathcal{2}$ of the time-line generation algorithm, which assigns the first available slot of the interval $\mathrm{I}, k P_{i} \leq I<(k+1) P_{i}, k=0,1, \ldots,\left(L C M / P_{i}-1\right)$ to connection $C_{i}$. 


\section{Time-line transition concepts}

\section{Comparison of the two time-lines}

We compare two time-lines by making reference to an instant $t_{l}$ called $t_{l c}$ situated in the present cycle of the running time-line: the two time-lines must be compared with the starting instants of their cycles which are coinciding. Call $L C M_{r}$ and $L C M_{n}$ the LCM of respectively the running and the new time-lines. We have two sub-cases:

- $L C M_{r} \leq L C M_{n}: t_{l c}$ belongs to the first cycle of the new time-line.

- $L C M_{r}>L C M_{n}$ : call $X=\frac{L C M_{r}}{L C M_{n}}$. We have $\mathrm{X}$ cycles of the new time-line during the present running time-line cycle. In a general way, $t_{l c}$ is such that $(b-$ 1). $L C M_{n} \leq t_{l c} \leq b \cdot L C M_{n}$, with $b=1,2, \ldots, X$, then $t_{l c}$ belongs to the $b^{\text {th }}$ cycle of the new time-line.

Definition 4.2 (Time-line transition instant) The instant of the time-line transition must guarantee the correctness on the slots generation, i.e. no slot duplications and no slot losses.

Theorem 4.1 If the time-line transition is made at the instant $t_{l c}$ where there are no pending connections, neither in the running time-line cycle nor in the $b^{\text {th }}$ cycle of the new time-line, then the time-line transition can be made and the correctness property is guaranteed.

\section{Proof}

Since at the instant $t_{l c}$ there is no pending connection in both time-lines, these means that slots have already been assigned for the current $P_{i}^{k}$ of every connection in both timelines. Then, even if the time-line is changed at this instant, as there were no pending connections in both time-lines, exactly one and only one slot will be assigned for every $P_{i}^{k+1}$ in the new time-line. Hence, correctness is guaranteed.

It is important to see that the simplest approach for changing the time-line with the correctness property is to make the time-line transition at the end of the running time-line cycle. However, this approach has a major drawback: it entails a high response time to users requests in the case of a long time-line cycle.

As previously mentioned, a high system responsiveness to users requests is highly desirable. Considering that connection periods can be long and can have different values, the time-line cycle can be extremely long. As a consequence, this approach may not provide the required system responsiveness.

In the following subsections we define an approach in order to provide a better system responsiveness than this one. The basic idea of the approach is to establish a condition which allows time-line transition before the end of the running time-line cycle. 


\section{Adding a connection}

We define a sufficient but not necessary condition for detecting the first instant $t_{l c}$ when there is no pending connections in both time-lines.

In order to guarantee the correctness, it is sufficient to respect the condition of no pending connections in both time-lines. The following lemma establish when it occurs.

Lemma $4.2 \forall t_{l c}$, if there is no pending connection in the new time-line at $t_{l c}$, then there is no pending connection in the running time-line at $t_{l c}$.

Refer to [CVJ94] for a proof of this lemma.

Finally, we define the transition instant $t_{l t}$ when the changing of the system time-line preserves the correctness.

Theorem 4.2 The transition instant $t_{l t}$ is the instant $t_{l c}$ which corresponds to the starting instant of the first unassigned slot in the new time-line.

\section{Proof}

It follows from lemma 4.1 that at the $t_{l t}$ instant there is no pending connection in the new time-line. By lemma 4.2, there is no pending connection in both time-lines. Hence, from theorem 4.1, the correctness is guaranteed.

We have represented in the figure 9.d the time-line change which guarantees the correctness.

\section{Removing a connection}

We have shown (theorem 4.1) that when there is no pending connections in both timelines, the correctness is guaranteed. Let us analyze when it occurs in the case of a connection is removed. We can easily see that the lemma 4.2 can be changed by making the permutation between running time-line and new time-line.

Lemma $4.3 \forall t_{l c}$, if there is no pending connection in the running time-line at $t_{l c}$, then there is no pending connection in the new time-line at $t_{l c}$.

Thus, we can define the transition instant $t_{l t}$.

Theorem 4.3 The transition instant $t_{l t}$ is the instant $t_{l c}$ which corresponds to the starting instant of the first unassigned slot in the running time-line.

\section{Proof}

It follows from lemma 4.1 that at the instant $t_{l t}$ there is no pending connection in the running time-line. By lemma 4.3 , there is no pending connection in both time-lines. Hence, from theorem 4.1, the correctness is guaranteed.

We have represented at figure $10 . d$ the time-line change which guarantees the correctness. 


\section{Time-line transition delay upper bound}

The worst-case delay to replace the running time-line by the new computed one, depends on the maximum number of consecutive assigned slots. We have shown [CVJ94] that this number is upper bounded by $\left(P_{n}-1\right) . P_{n}$ being the longest period of the connection period set.

\section{CONCLUSION}

The study presented in this paper is, in our mind, an innovative work in the area of real-time isochronous services based on the PA access of a DQDB network:

- we have specified a service-protocol pair for the bandwidth allocation and releases phases, which has procedures similar to the procedures for originating and terminating circuit switched calls. Furthermore, this service-protocol pair has been formally modeled with Petri nets and validated.

- we have proposed a bandwidth allocation scheme which consists of an off line scheduling algorithm and an on line mode change algorithm. The off line scheduling algorithm which is a discrete version of the well known Rate Monotonic algorithm is easy to be implemented. The on line mode change algorithm which is, to our best knowledge, a new contribution is independent of the utilization context.

Finally, we also plan to make a performance evaluation of the service-protocol pair specified for the connection-oriented isochronous service by using the methodology that have been proposed in [CJ94].

\section{References}

[BRA83] G. W. BRAMS. Réseaux de Petri. Masson, Paris., 1983.

[C.J94] Rosa M. L. R. Carmo and Guy Juanole. Modeling and Evaluating the DQDB Protocol with Stochastic Timed Petri Nets. In Proceedings of the $2^{\text {nd }}$ International Workshop on Modeling, Analysis, and Simulation of Computer and Telecommunications Systems (MASCOTS 94), pages 269-275, Durham, North Carolina, USA, january 1994.

[CVJ94] Rosa L. R. Carmo, Francisco Vasques, and Guy Juanole. Real-Time Communication Services in a DQDB Network. Technical Report, LAAS du CNRS, 1994.

[IEE90] IEEE. Distributed Queue Dual Bus (DQDB) subnetwork of a Metropolitan Area Network (MAN). Standard P802.6/D15, IEEE, 1990.

[LL73] C. L. Liu and James W. Layland. Scheduling Algorithms for Multiprogramming in a Hard Real-Time Environment. Journal of the Association for Computing Machinery, 20(1):46-61, 1973. 
[Llo90] J.C. Lloret. Réseaux Prédicat Transition étiquetés pour la modélisation et la vérification des systèmes informatiques répartis. $\mathrm{PhD}$ thesis, Université Paul Sabatier de Toulouse, 1990.

[MB92] Biswanath Mukherjee and Chatschik Bisdikian. A journey through the DQDB network literature. Performance Evaluation, 165:129-158, 1992.

[Mil80] R. Milner. A Calculus of Communicating Systems. In Lecture Notes in Computer Science, volume 92, Berlin Heidelberg, 1980. Springer-Verlag.

[TGS91] P. Tran-Gia and Th. Stock. Modelling of the DQDB Access Protocol and ClosedForm Approximation. In High-Capacity Local and Metropolitan Area Networks, pages 253-265. Springer Verlag, 1991.

Guy Juanole received the "Doctor es Sciences" degree from the University Paul Sabatier, Toulouse, France, 1978. He is, at the present time, Professor at the University Paul Sabatier (lectures in Automatic Control, Communications Networks and Formal Models) and Researcher at the Laboratory LAAS (Laboratoire d'Analyse et d'Architectures des Systemes) of the CNRS (Centre National de la Recherche Scientifique) in Toulouse. His research interests are in the area of the Petri nets based models with a major emphasis on the Timed and Stochastic Petri nets and their applications to communication networks and protocols (high-speed networks, industrial networks).

Rosa L. R. Carmo received the Ph.D. degree from the University Paul Sabatier, Toulouse, France, 1994. She is Researcher at the Federal University of Rio de Janeiro, Brazil. Her research interests are in the area of performance analysis and modeling of high speed networks, network traffic characterization and multimedia computing and communication.

Francisco Vasques is Assistant Professor at the University of Porto, Portugal, and is currently on leave at LAAS-CNRS, preparing a Ph.D. degree. His main research interests include scheduling algorithms, communication protocols and fault-tolerant issues within distributed real-time systems. 